US Army Corps

of Engineers $S_{\circledast}$

Engineer Research and

Development Center

Geometric-Acoustics Analysis of Singly Scattered, Nonlinearly Evolving Waves by Circular Cylinders

Michael B. Muhlestein and Carl R. Hart

October 2020

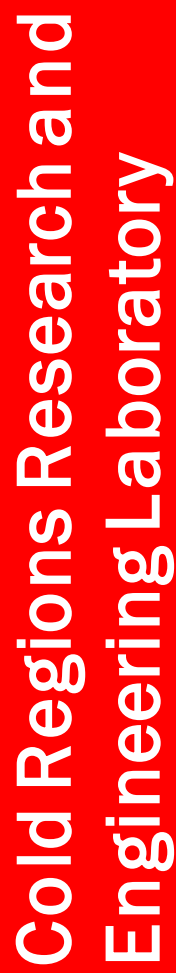


The U.S. Army Engineer Research and Development Center (ERDC) solves the nation's toughest engineering and environmental challenges. ERDC develops innovative solutions in civil and military engineering, geospatial sciences, water resources, and environmental sciences for the Army, the Department of Defense, civilian agencies, and our nation's public good. Find out more at www.erdc.usace.army.mil.

To search for other technical reports published by ERDC, visit the ERDC online library at https://erdclibrary.on.worldcat.org/discovery. 


\section{Geometric-Acoustics Analysis of Singly Scattered, Nonlinearly Evolving Waves by Circular Cylinders}

Michael B. Muhlestein and Carl R. Hart

Cold Regions Research and Engineering Laboratory

U.S. Army Engineer Research and Development Center

72 Lyme Road

Hanover, NH 03755

Final report

Approved for public release; distribution is unlimited.

Prepared for US Army Corps of Engineers

Washington, DC 20314-1000

Under Research, Development, Test, and Evaluation Program Element 611102T2400 


\section{Preface}

This study was conducted for the US Army Corps of Engineers, Office of the Assistant Secretary of the Army for Acquisition, Logistics, and Technology [ASA(ALT)] under Research, Development, Test, and Evaluation Program Element 611102T2400, Project Number AB2, AMSCO Code 611102AB200. The technical monitor was Mr. Ritchie L. Rodebaugh, Technical Director for Geospatial Research and Engineering.

The work was performed by the Signature Physics Branch of the Research and Engineering Division, U.S. Army Engineer Research and Development Center, Cold Regions Research and Engineering Laboratory (ERDCCRREL). At the time of publication, Dr. M. Andrew Niccolai was Branch Chief and Mr. J.D. Horne was Division Chief. The Deputy Director of ERDC-CRREL was Mr. David B. Ringelberg, and the Director was Dr. Joseph L. Corriveau.

This Miscellaneous Paper (MP) was originally published in the Journal of the Acoustic Society of America, June 2020.

The Commander of ERDC was COL Teresa A. Schlosser and the Director was Dr. David W. Pittman. 


\title{
Geometric-acoustics analysis of singly scattered, nonlinearly evolving waves by circular cylinders
}

\begin{abstract}
:
Geometric acoustics, or acoustic ray theory, is used to analyze the scattering of high-amplitude acoustic waves incident upon rigid circular cylinders. Theoretical predictions of the nonlinear evolution of the scattered wave field are provided, as well as measures of the importance of accounting for nonlinearity. An analysis of scattering by many cylinders is also provided, though the effects of multiple scattering are not considered. Provided the characteristic nonlinear distortion length is much larger than a cylinder radius, the nonlinear evolution of the incident wave is shown to be of much greater importance to the overall evolution than the nonlinear evolution of the individual scattered waves.
\end{abstract}

\section{INTRODUCTION}

The scattering of impulsive high-amplitude waves with high frequency content by rigid objects is an important prob-lem in studying the effects of shock waves on natural and arti-ficial structures. An example of such a scenario would be sonic boom propagation through a forested area. While the scattering of linear, or low-amplitude, waves by single and multiple scatterers is a well-developed field with an extensive literature (see, e.g., Refs. 1-4), less is known about the effects of scatter-ing on nonlinear waves. Abbasov studied the generation of sec-ond harmonics of initially sinusoidal waves incident upon a rigid cylinder. ${ }^{5}$ In addition, many papers associated with the scattering of sound by sound in the presence of obstacles have analyzed the generation of sum and difference frequencies of multiple acoustic beams incident on rigid objects using both experimental and analytical means (e.g., Refs. 6 and 7).

Geometric acoustics, or acoustic ray theory, has been used extensively to model linear ${ }^{8}$ sound propagation with and without diffraction, and also nonlinear ${ }^{8-11}$ sound propagation in the absence of diffraction. Perhaps the most common application of geometric acoustics has been in the study of sound propagation through domains with slowly varying material properties, such as the atmosphere or the ocean. ${ }^{8,12}$ Ray theory has also been used to study the scattering of linear waves by discrete scatterers. ${ }^{13}$ To the authors' knowledge, no study incorporates nonlinearity in geometric scattering of sound from discrete rigid scatterers. Exploring this topic is the purpose of this paper.

The outline of the paper is as follows: The theoretical background and development of the geometric scattering of nonlinear waves is presented in Sec. II with special emphasis on the scattering of sound by rigid circular cylinders. Section III briefly considers the case of nonlinear waves incident upon many arbitrarily placed rigid circular cylinders, and finally Sec. IV provides a summary.

\section{THEORETICAL DEVELOPMENT}

\section{A. Existing results}

Geometric-acoustics theory is based on the asymptotic limit of infinitesimally small wavelengths, and are reasonably valid for $\lambda \ll a$, where $\lambda$ is the wavelength and $a$ is the smallest characteristic size of the propagation domain (e.g., the radius of a single infinite cylinder in an otherwise free space environment). In this limit, diffraction does not affect wave propagation and any wave front may be well approximated as locally planar, which greatly simplifies scattering analyses, as shown below.

A review of the application of geometric acoustics to nonlinear wave propagation is given by Morfey et al. ${ }^{11}$ Following their notation, the arrival phase along a ray tube in a lossless homogeneous medium may be written as

$$
\tau=\tau_{0}-\frac{\beta}{\rho_{0} c_{0}^{3}} \zeta p_{\text {ref }}(\tau),
$$

where $\tau$ is the retarded time of arrival, $\tau_{0}$ is the retarded time of arrival if nonlinearity were neglected, $\beta$ is the coefficient of nonlinearity, $\rho_{0}$ is the ambient mass density, $c_{0}$ is the small-signal sound speed, $\zeta$ is a modified distance, and $p_{\text {ref }}$ is the acoustic pressure at the reference location. The modified distance may be written as

$$
\zeta=\int_{0}^{s} \sqrt{\frac{A(0)}{A\left(s^{\prime}\right)}} d s^{\prime},
$$

where $A$ is the differential area of the ray tube as a function of the ray path distance $s$. The acoustic pressure amplitude at a particular phase is assumed to decay only by either 
nonlinear attenuation at shocks or as the differential area decreases, such that the quantity

$$
q(\tau) \equiv \sqrt{\frac{A(s)}{A(0)}} p_{\text {ref }}\left(\tau_{0}\right)
$$

remains constant for all $s$ (away from shocks). These equations turn out to be solutions to the partial differential equation $^{14}$

$$
\frac{\partial q}{\partial \zeta}=\frac{\beta}{\rho_{0} c_{0}^{3}} q \frac{\partial q}{\partial \tau},
$$

which is the inviscid Burgers equation that describes weakly nonlinear acoustic one-way propagation in one spatial dimension (here $\zeta$ ). These results are accurate up to quadratic nonlinearity [i.e., neglecting terms that are $O\left(\epsilon^{3}\right)$, where $\epsilon$ is the acoustic Mach number] and, in particular, allow for the generation of shocks. The shock formation distance of a time-harmonic signal with initial angular frequency $\omega$ and amplitude $p_{0}$ occurs at

$$
\zeta=\bar{\zeta} \equiv \frac{\rho_{0} c_{0}^{3}}{\beta \omega p_{0}} .
$$

Modeling the propagation of shocks requires additional constraints, such as weak shock theory. ${ }^{14} \mathrm{~A}$ full-wave analysis of nonlinear wave propagation that accounts for diffraction results in a much more complicated equation than Eq. (4), such as the Khokhlov-Zabolotskaya-Kuznetsov equation. ${ }^{15}$ Thus, the neglect of diffraction leads to significant simplification.

Geometric scattering of linear waves by curved structures was described by Pierce. ${ }^{8}$ To summarize, the scattering of acoustic rays from a rigid curved structure in an otherwise homogeneous medium may be described by the reflected wave:

$$
p_{r}\left(\vec{x}_{s}+\hat{n}_{r} l, t\right)=\sqrt{\frac{A(0)}{A(l)}} p_{i}\left(\vec{x}_{s}, t-l / c_{0}\right),
$$

where $p_{r}$ is the scattered pressure wave, $p_{i}$ is the incident pressure wave, $\vec{x}_{s}$ is the position vector of the reflection point of the ray path, $\hat{n}_{r}$ is the unit direction vector of the reflected wave, and $l$ is the distance of the reflected wave from the scattering site (equivalent to $s$ with $s=0$ the scattering site). Equation (6) is valid for general scattering problems, but here the analysis will be restricted to the simple case of an incident plane wave propagating in the $-\hat{x}$ direction and incident upon a circular cylinder of radius $a$ located at an arbitrary point and extending in the $z$ direction. In this simple case, shown schematically in Fig. 1, the scattered direction vector and ratio of areas may be written as

$$
\begin{aligned}
& \hat{n}_{r}=\cos \left(2 \theta_{s}\right) \hat{x}+\sin \left(2 \theta_{s}\right) \hat{y}, \\
& \frac{A(l)}{A(0)}=1+\frac{2 l}{a \cos \theta_{s}},
\end{aligned}
$$

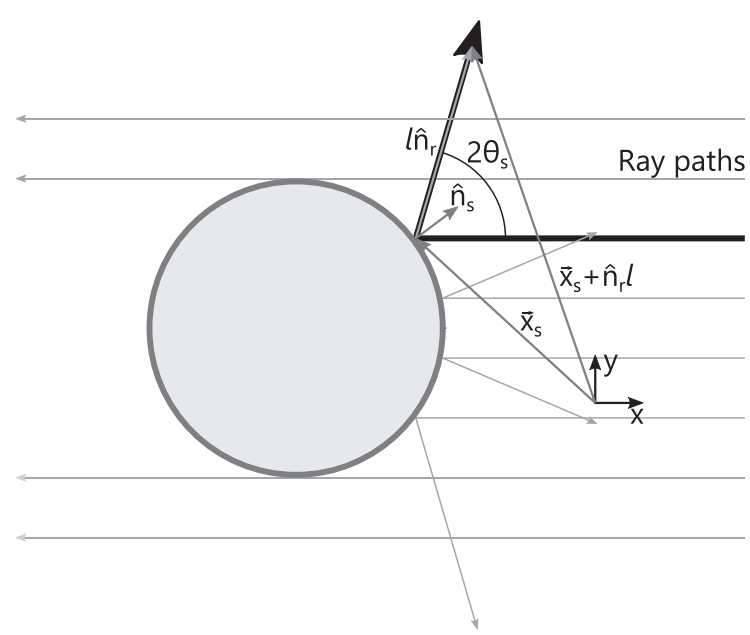

FIG. 1. Schematic showing the ray paths of a plane wave incident upon a rigid cylinder of radius $a$ from the positive $x$ direction.

where $\theta_{s}$ is the angle at which the scattering occurs. Note that the direction of the reflected wave may be written as $\hat{n}_{r}=\cos (\theta) \hat{x}+\sin (\theta) \hat{y}$, where $\theta=2 \theta_{s}$. Another benefit to only considering cylinders with circular cross sections is that the scattered field will only diverge and so caustics are avoided. The presence of caustics requires additional considerations. ${ }^{11}$

\section{B. Geometric scattering of nonlinear waves}

As with the linear case, the scattering problem divides into three parts-prior to the scattering event, the scattering event itself, and after the scattering event. The incident wave is assumed to be planar for simplicity; other geometric spreading behaviors could have been chosen using Eq. (4). The acoustic pressure in each ray of the incident wave may then be modeled using the inviscid Burgers equation for a plane wave [equivalent to Eq. (4) with $\zeta=x_{0}-x$ and $x_{0}$ being the source location]

$$
\frac{\partial p}{\partial(-x)}=\frac{\beta}{\rho_{0} c_{0}^{3}} p \frac{\partial p}{\partial \tau}
$$

and has the uniform shock formation distance of

$$
\bar{x}=\frac{\rho_{0} c_{0}^{3}}{\beta \dot{p}_{\max }},
$$

where $\dot{p}_{\max }$ is the maximum rate of change of the acoustic pressure at the origin. After the scattering event each ray path propagates independently (which is a basic assumption of geometric acoustics) by Eq. (4) with Eqs. (2) and (8) yielding

$$
\zeta=\int_{0}^{l} \sqrt{\frac{1}{1+2 l^{\prime} / a \cos \theta_{s}}} d l^{\prime}=a \cos \theta_{s}\left(\sqrt{1+\frac{2 l}{a \cos \theta_{s}}}-1\right) .
$$

The shock formation distance (as measured from the scattering site) in the modified length system remains the same as 
for the plane wave $\bar{\zeta}=\bar{x}$. Inverting the definition of $\zeta$ for $l$, the shock formation distance in $l$ may be written as

$$
\bar{l}=\bar{x}+\frac{\bar{x}^{2}}{2 a \cos \theta_{s}} .
$$

The scattering event itself occurs in a very thin boundary layer around the scattering object, and is assumed to be accurately described by linear theory. Nonlinear phenomena that are not taken into account include radiation pressure, acoustic streaming, and nonlinear absorptive processes. Since these local nonlinear phenomena are typically assumed to be third-order or higher effects in terms of the acoustic Mach number $\epsilon$, this assumption limits the realm of applicability of this theory to weakly nonlinear propagation. Assuming that $\epsilon^{3}=0.1$ is an appropriate upper limit for the present assumption, the maximum amplitude in air that may be described is about $190 \mathrm{~dB}$ re $20 \mu \mathrm{Pa}$. All structures considered in this paper are assumed to be rigid, and so perfect reflection is observed.

While the shock formation distance is the classic nonlinear length scale it may not be the most useful in all cases. For example, the incident wave may already contain a shock, in which case the shock formation distance is simply 0 . Alternative lengths may be used for $\bar{x}$, such as $\rho_{0} c_{0}^{3} T_{0} / \beta p_{0}$, where $T_{0}$ is a characteristic time scale, without changing the nature of the analysis presented here. For simplicity, focus will be given to the time-harmonic shock formation distance.

An explanation of the physical significance of the modified length $\zeta$ is helpful. Equation (4) suggests that the behavior of the waveform along any reflected ray behaves exactly as a one-dimensional plane wave would with the two exceptions that the physical propagation distance ( $l$ for the present case) should be replaced by the modified length $\zeta$ and that the acoustic pressure should be replaced by the modified acoustic pressure $q$. This means that at the point where $\zeta=\rho_{0} c_{0}^{3} / \beta \dot{q}_{\max } \equiv \bar{\zeta}$ shocks will form. (Since $\dot{q}_{\max }$ is calculated at the origin, or the scattering site, it is identical to $\dot{p}_{\text {max }}$.) Furthermore, if the source waveform is a sinusoid then at the point where $\zeta$ reaches $3 \bar{\zeta}$ the waveform will assume a full sawtooth shape. ${ }^{14}$ Thus $\zeta$ may be considered a measure of the nonlinear distortion regardless of the geometric spreading of the rays. These statements are not true for the physical distance $l$ : e.g., at $l=\rho_{0} c_{0}^{3} / \beta \dot{p}_{\max }$ shocks will not have formed unless the propagation is strictly planar.

A graphical explanation of the meaning of the modified length $\zeta$ is shown in Fig. 2, where $\zeta$ is shown as a function of the true physical distance for scattering angles $\theta=0^{\circ}, 60^{\circ}, 120^{\circ}$, and $178^{\circ}$. Note that for $\theta=180^{\circ}$ the amplitude goes to zero and so is parallel to the horizontal axis, and so $178^{\circ}$ was chosen to represent the near-forwardscattering direction; see below for more discussion. In this particular plot the planar shock formation distance $\bar{x}$ was chosen to be $2 a$, where $a$ is the cylinder radius. Plots of the modified length assuming perfect planar propagation (identical to physical distance), uniform cylindrical propagation,

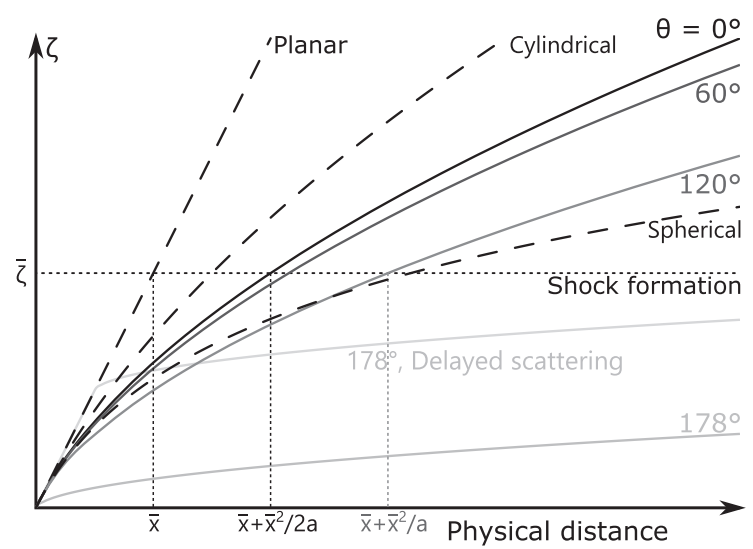

FIG. 2. The modified length $\zeta$ as a function of the true physical distance for scattering angles $\theta=0^{\circ}, 60^{\circ}, 120^{\circ}$, and $178^{\circ}$. See the main text for a full description.

and uniform spherical propagation are also shown for Ref. 14. Shock formation occurs as $\zeta$ reaches the dotted horizontal line. The physical distances where shock formation occurs are marked for planar propagation, $\theta=0^{\circ}$, and $\theta=120^{\circ}$. With one exception, " $178^{\circ}$, Delayed scattering," all of the modified lengths shown assume scattering occurs at the origin of the plot (bottom left corner). The delayed scattering curve assumes planar propagation continues until half-way to shock formation in planar propagation, and then follows the scattering angle of $\theta=178^{\circ}$.

It is apparent that the planar propagation reaches shock formation first. The modified length curves for the scattered rays are all cylindrical but the smaller scattering angles evolve faster than the higher angles, leading to more rapid formation of shocks. The spherical case is logarithmic in nature rather than a power law, ${ }^{14}$ and therefore in the far field will evolve slower than any of the scattered waves. Looking forward to analyzing the impact of scatterers located at many locations, the case where the scattering event is delayed to a farther point is also of interest. The overall evolution of this scattered ray is advanced by a fixed amount relative to the same scattered ray from the origin due to the extended evolution of the incident planar wave.

\section{The importance of nonlinearity after scattering}

A natural question is whether it is important to account for the nonlinear evolution for a given ray path. A relevant metric is the ratio of the planar shock formation distance to the ray shock formation distance $\bar{x} / \bar{l}$, which may be interpreted as the degree to which nonlinearity is important after the scattering relative to how important it was prior to the scattering. Alternatively, the ratio may be interpreted as the importance of nonlinearity relative to the geometric spreading of the scattered wave, with $\bar{x} / \bar{l}=1$ indicating that nonlinearity dominates spreading and $\bar{x} / \bar{l}=0$ indicating that spreading dominates nonlinearity.

A polar plot of $\bar{x} / \bar{l}$ is shown in Fig. 3 for five values of $\bar{x} / a$. For small values of $\bar{x} / a$ (shock formation distance small relative to the size of the scattering object) the ratio 


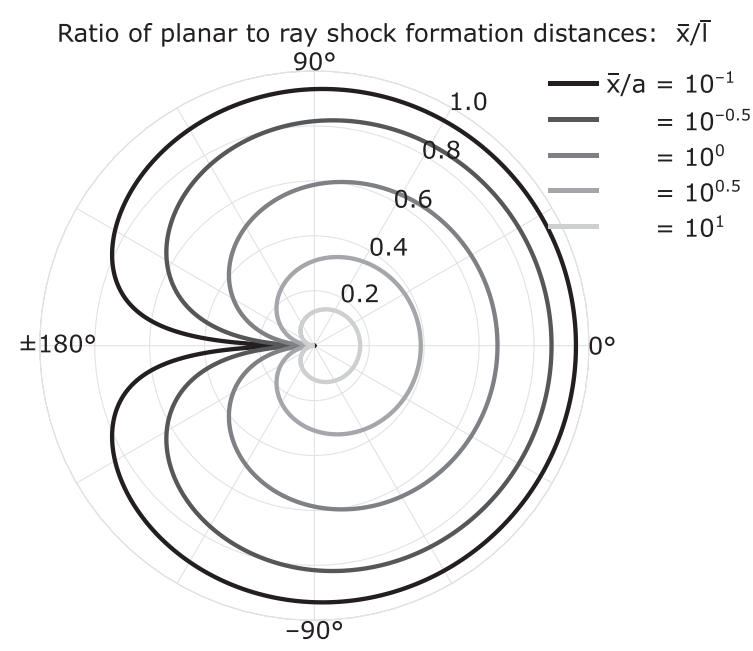

FIG. 3. Ratio of the planar shock formation distance to the ray shock formation distance $\bar{x} / \bar{l}$ as a function of scattering angle for several values of $\bar{x} / a$.

$\bar{x} / \bar{l}$ is only slightly less than 1 for most scattering angles, meaning that nonlinearity is almost as important after the scattering event as before and therefore dominates geometric spreading as the most important phenomenon. The reason nonlinearity dominates in this case is that the shock formation distance is small enough relative to the cylinder radius that the cylinder surface may be approximated as a plane, and very little energy has been lost to geometric spreading. On the other hand, large values of $\bar{x} / a$ lead to relatively small values of $\bar{x} / \bar{l}$ for all angles, meaning that nonlinearity is much less important after the scattering event than before. The reason for this loss of importance is that the wave amplitude decays with the cylindrical spreading, leading to less wave distortion.

The importance of nonlinearity remains fairly constant as a function of $\theta$ for $|\theta|<60^{\circ}$, but for larger values of $\bar{x} / a$ the importance starts to decay for $|\theta|>60^{\circ}$. In the limit of $\bar{x} / a \rightarrow \infty$ (i.e., the limit that nonlinearity may be completely neglected), the relative importance of nonlinearity approaches the form $\bar{x} / \bar{l} \rightarrow 2 \cos (\theta / 2) /(\bar{x} / a)$. Thus, side and forward-scattering is less impacted by nonlinearity than back-scattering.

It is worth noting that in the exact forward-scattering direction, $180^{\circ}$, all of the ratios of $\bar{x} / \bar{l}$ go to zero, suggesting that nonlinearity is not important in this direction. However, this result is misleading for two reasons. First, the geometric acoustics approximation of no diffraction becomes very poor in the shadow region of the cylinder except in extremely high-frequency cases, and so the predictions close to $\theta=180^{\circ}$ are inaccurate. (This point may be proven in the linear limit using standard Fresnel approximations; see, e.g., Ref. 16.) Second, a tacit assumption used by analyzing the reflected wave is that wave superposition holds, meaning the total acoustic pressure field may be written as $p=p_{i}+p_{r}$. The angles for which this assumption is poor may be estimated by approximating the reflected wave fronts as being perfectly cylindrical about the center of the scatterer. Then for a pulse of width $L$ and a distance $r$ from the scatterer the incident and scattered waves will overlap (and the assumption of superposition becomes poor) for all $\theta \in\left\{180^{\circ}-\cos ^{-1}(1-L / r), 180^{\circ}+\cos ^{-1}(1-L / r)\right\}$. Far from the scatterer where $L / r \ll 1$ the inverse cosine term may be approximated as $\sqrt{2 L / r} \mathrm{rad}$, and so the angular overlap region asymptotically approaches zero at $180^{\circ}$.

Finally, the patterns of the ratio of the planar shock formation distance to the ray shock formation distance will vary and likely be more complicated for non-circular cylinders and other scattering bodies. This simple analysis is not likely to be accurate if caustics appear in the reflected field.

\section{Waveform evolution of the scattered wave}

The simplifications of geometric acoustics allow one to use standard methods, such as the Poisson solution, ${ }^{14}$ to describe the nonlinear evolution of the scattered field in the time domain. Using the time domain means that all harmonics are simultaneously accounted for. This section analyzes the variation in the waveform evolution along different scattered ray paths for an example impulsive source condition.

Consider a planar Gaussian-derivative pressure pulse (chosen to easily exhibit the effects of nonlinear distortion prior to shock formation, and not to represent any particular physical source) normally incident upon a cylinder with radius $a=10 \mathrm{~cm}$. The ambient medium is assumed to be air with a mass density given by $\rho_{0}=1.201 \mathrm{~kg} / \mathrm{m}^{3}$, smallsignal sound speed by $c_{0}=343 \mathrm{~m} / \mathrm{s}$, and coefficient of nonlinearity by $\beta=1.4$. The pressure wave as measured at the origin without the cylinder present may be described by the function

$$
p=-p_{0}\left(t / T_{0}\right) e^{-\left(t / T_{0}\right)^{2}}
$$

with $p_{0}=200 \mathrm{~Pa}$ and $T_{0}=0.01 a / c_{0} \approx 2.9 \mu \mathrm{s}$. The peak frequency of this function is $f=1 / 2^{3 / 2} \pi^{2} T_{0} \approx 12.2 \mathrm{kHz}$. While any finite value of $T_{0}$ will realistically lead to diffraction effects at some distance, geometric acoustics provides an estimate of the asymptotic behavior for $k a \gg 1$, where $k$ $=2 \pi f / c_{0}$ is a representative wavenumber. For this scenario $k a \approx 7720 \gg 1$, thus justifying the use of geometric acoustics. Regardless, the results described below should not be interpreted as the precise waveforms expected to be measured, but rather guides for how to interpret measured waveforms.

For simplicity the nonlinear evolution of the incident wave as it propagates from one side of the cylinder to the other is neglected. This approximation is valid if the diameter of the cylinder is much smaller than the shock formation distance. The shock formation distance of the incident wave may be written as

$$
\bar{x}=\frac{\rho_{0} c_{0}^{3}}{\beta \dot{p}_{\max }}=\frac{\rho_{0} c_{0}^{3} T_{0} e^{3 / 2}}{2 \beta p_{0}} \approx 113 \mathrm{~cm}
$$

which leads to $\bar{x} / a \approx 11$. A discussion about the importance of the evolution of the incident wave is presented in Sec. III below. The shock formation distances of the ray paths differ from each other after scattering, as described above. 
The shock formation distances of the ray paths at specific angles may be calculated using Eq. (12). Table I summarizes the shock formation distances for $\theta=2 \theta_{s}=0,30^{\circ}, 60^{\circ}, 90^{\circ}$, $120^{\circ}, 150^{\circ}$, and $178^{\circ}$. As is clear from the table, the shock formation distance increases with increasing scattering angle, and starts approaching infinity as $\theta \rightarrow 180^{\circ}$. This increasing shock formation distance is associated with a decreasing importance of nonlinearity in the propagation, as shown by the diminishing values of the plane to ray shock formation distance ratio, also shown in Table I.

Predicted waveforms of the scattered field of the pulse as a function of the retarded time for the seven angles described in Table I are shown in Fig. 4 both accounting for nonlinearity and neglecting it. The distance from the origin at which the waveforms were predicted is $100 a$ (approaching the $l / a \rightarrow \infty$ limit), or $10 \mathrm{~m}$. The waveforms have been aligned such that they all cross zero at $t=0$ for the sake of comparison. The most significant difference between the linear and nonlinear predictions occurs for the $\theta=0$ case, where the peak amplitude of the nonlinear case arrives $0.012 a / c_{0} \approx 3.5 \mu$ s before the linear case, and a shock has formed in the nonlinear case. As $\theta$ increases the difference in arrival times between the linear and nonlinear cases decreases, and at $\theta=178^{\circ}$ the difference is barely perceptible at $0.002 a / c_{0} \approx 0.5 \mu \mathrm{s}$. The amplitude of the scattered waveforms decrease with increasing scattering angle, but does not change when nonlinearity is accounted for versus the linear cases. However, if the measurement location was well beyond the shock formation distance of the ray paths with lower scattering angles (not shown here) the resulting shocks and the associated excess attenuation would decrease the observed amplitude of the nonlinear cases.

\section{SCATTERING FROM MANY CYLINDERS}

Many important applications are associated with sound scattering by not just one but many obstacles. This section considers the scattering of an incident plane wave by a collection of identical rigid circular cylinders of radius $a$. Since the amplitude of the scattered waves decays rapidly it is often appropriate to assume that after two or more scattering events the propagation becomes essentially linear. To focus on the impact of nonlinearity on the scattered field only the single-scattered field is considered. The centers of the cylinders are chosen to be located at $\vec{x}_{i}=\left(x_{i}, y_{i}\right)$ for $i \in 1$, $2, \ldots, N$, where $N$ is the number of cylinders, and the measurement location is at $\vec{x}_{0}=\left(x_{0}, y_{0}\right)$ (see Fig. 5 for a schematic). The distance from the cylinder centers to the

TABLE I. Summary of ray shock formation distances (rounded to nearest meter) and the ratio of the planar shock formation distance to the ray shock formation distances for several scattering angles. The incident plane wave is a Gaussian pulse with a shock formation distance of approximately $1.1 \mathrm{~m}$.

\begin{tabular}{llllllll}
\hline \hline Scattering angle & $0^{\circ}$ & $30^{\circ}$ & $60^{\circ}$ & $90^{\circ}$ & $120^{\circ}$ & $150^{\circ}$ & $178^{\circ}$
\end{tabular}

Ray shock formation distance $7.5 \mathrm{~m} 7.7 \mathrm{~m} 8.5 \mathrm{~m} 10 \mathrm{~m} 14 \mathrm{~m} 26 \mathrm{~m} 368 \mathrm{~m}$ Plane to ray ratio $\bar{x} / \bar{l}$ $\begin{array}{llllllllll}0.150 & 0.146 & 0.133 & 0.111 & 0.081 & 0.044 & 0.003\end{array}$

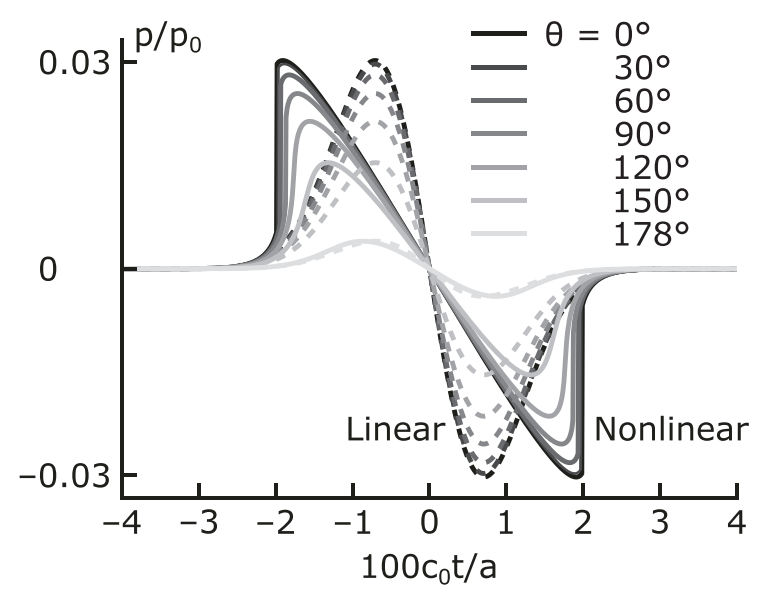

FIG. 4. Predicted scattered waveforms for a planar Gaussian-derivative incident pulse at several angles as a function of time. The waveforms are associated with signals measured $r=100 a$ from the origin, where $a$ is the radius of the cylinder. The acoustic pressure $p$ waveforms are normalized by the incident pulse amplitude $p_{0}$ and the time is normalized by the cylinder radius and the wavespeed $c_{0}$. The arrival times have been aligned such that the waveforms cross zero at $t=0$.

measurement location is denoted by $R_{i}$ $=\sqrt{\left(x_{i}-x_{0}\right)^{2}+\left(y_{i}-y_{0}\right)^{2}}$. The present analysis is restricted to the limiting case of a dilute concentration of scatterers, or $\hat{d} \gg a$, where $\hat{d}$ is the smallest characteristic separation distance between cylinders, meaning that shadowing effects of cylinders closer to the measurement location on the reflected waves of the farther cylinders may be ignored. Since the overall amplitudes of the scattered waves depend almost entirely on linear acoustics, this section focuses on the observed nonlinear distortion of the scattered field rather than on waveform amplitudes.

From Eq. (6) the scattered wave measured associated with cylinder $i$ may be written as

$$
p_{r, i}\left(\vec{x}_{s, i}+\hat{n}_{r, i} l_{i}, t\right)=\sqrt{\frac{A(0)}{A\left(l_{i}\right)}} p_{i}\left(\vec{x}_{s, i}, t-l_{i} / c_{0}\right) .
$$

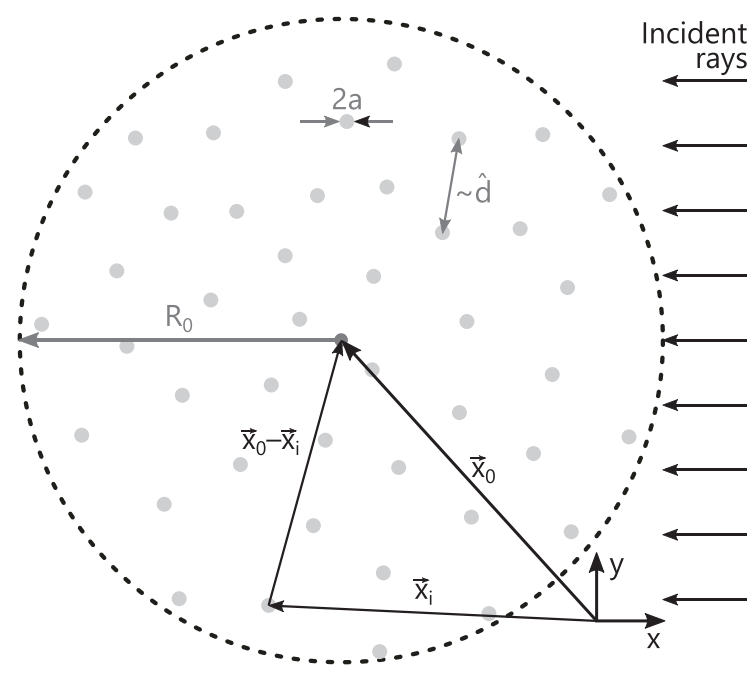

FIG. 5. Schematic of the scattering geometry for many identical cylinders of radius $a$ within a region of radius $R_{0}$ with a characteristic spacing of $\hat{d}$. 
Since the measurement site is at $\vec{x}_{0}$ and the scattering site $\vec{x}_{s, i}=\vec{x}_{i}+\vec{x}_{s}$ the relative position vector may be written as

$$
\vec{x}_{0}-\vec{x}_{i}=\vec{x}_{s}+\hat{n}_{r, i} l_{i}
$$

and so

$$
R_{i}^{2}=a^{2}+l_{i}^{2}+2 a l_{i}\left(\cos \theta_{s, i} \cos \theta_{i}+\sin \theta_{s, i} \sin \theta_{i}\right),
$$

where $\theta_{s, i}$ is the angle at which the incident wave strikes the cylinder and $\theta_{i}$ is the scattering angle. From above, $\theta_{i}=2 \theta_{s, i}$. The scattering angle satisfies the condition

$$
\tan \theta_{i}=\frac{y_{i}+a \sin \theta_{i} / 2-y_{0}}{x_{i}+a \cos \theta_{i} / 2-x_{0}} .
$$

In the limit $l_{i} \gg a$ the propagation distance may be approximated as

$$
l_{i} \approx R_{i}-a \cos \left(\theta_{i} / 2\right),
$$

and that the scattering angle $\theta_{i}$ satisfies

$$
\tan \theta_{i} \approx \frac{y_{i}-y_{0}}{x_{i}-x_{0}} .
$$

As above the modified length may be identified as

$$
\zeta_{i}=a \cos \theta_{s}\left(\sqrt{1+\frac{2 l}{a \cos \theta_{s}}}-1\right) \approx \sqrt{2 R_{i} a \cos \left(\theta_{i} / 2\right)} .
$$

Thus, the approximate nonlinear phase [from Eq. (1)] may be written as

$$
\phi_{i} \approx t-\frac{R_{i}}{c_{0}}+\frac{a}{c_{0}} \cos \left(\theta_{i} / 2\right)+\frac{\beta}{\rho_{0} c_{0}^{3}} \zeta_{i} p_{i} .
$$

For sufficiently short pulses, each of the arrivals are not likely to interact with each other, and so the total scattered field measured may be approximated by linear superposition

$$
p_{r}\left(\vec{x}_{0}, t\right) \approx \sum_{i} p_{r, i}\left(\vec{x}_{0}, t\right) .
$$

Linear superposition of the nonlinear case may also be justified because the propagation direction is typically different from each cylinder, and so the overlapping domain is small enough to neglect nonlinear effects.

A number of statistics may be calculated at this point. One is the average modified length, which may be written as

$$
\begin{aligned}
\langle\zeta\rangle & =\frac{\int_{\Omega} w(R, \theta) \zeta(R, \theta) d \Omega}{\int_{\Omega} w(R, \theta) d \Omega} \\
& \approx \frac{\int_{\Omega} w(R, \theta) \sqrt{2 a R \cos (\theta / 2)} d \Omega}{\int_{\Omega} w(R, \theta) d \Omega},
\end{aligned}
$$

where $\Omega$ is the domain of the scatterers and $w$ is the number density of scatterers at each given value of $R$ and $\theta$. The average modified length is a characteristic measure of the nonlinear distortion one would measure from several scattering sites. Notice that the subscript $i$ has been dropped from $\zeta, R$, and $\theta$ as the analysis has transitioned from discrete to continuous. For the case of uniformly distributed scatterers (randomly or otherwise) where $w(R, \theta)=w_{0}$ in a circle of radius $R_{0}$ around the measurement point with a density sufficiently high that a continuum approximation is appropriate, $\langle\zeta\rangle$ may be approximated as

$$
\begin{aligned}
\langle\zeta\rangle & \approx \frac{2}{\pi R_{0}^{2}} \int_{0}^{R_{0}} \int_{0}^{\pi} \sqrt{2 a R \cos (\theta / 2)} R d \theta d R \\
& =\frac{32 \sqrt{\pi a R_{0}}}{5 \Gamma^{2}(1 / 4)} \approx 0.863 \sqrt{a R_{0}},
\end{aligned}
$$

where $\Gamma$ is the Gamma function. The standard deviation is given by

$$
\begin{aligned}
\sigma_{\mathrm{sd}}(\zeta) & \approx \sqrt{\frac{2}{\pi R_{0}^{2}} \int_{0}^{R_{0}} \int_{0}^{\pi}(\sqrt{2 a R \cos (\theta / 2)}-\langle\zeta\rangle)^{2} R d \theta d R} \\
& =\sqrt{\frac{8 a R_{0}}{75 \pi}\left(25-\frac{384 \pi^{2}}{\Gamma^{4}(1 / 4)}\right)} \approx 0.323 \sqrt{a R_{0}}
\end{aligned}
$$

Other geometries of interest may also be considered.

Perhaps more important than just the modified length, which is from the scatterer to the observer, is the total modified propagation path. This distance is the distance the incident wave propagates to get to the scatterer plus the modified path length from the scatterer to the measurement location. If the incident wave measured at the center of the above-described circle of scatterers is assumed to have a propagation path length of 0 , where scattering sites with positive values of $x$ have negative propagation paths for the incident wave, then the average propagation path may be expressed as

$$
\left\langle\zeta_{\text {tot }}\right\rangle=\langle\zeta-R \cos (\theta)\rangle=\langle\zeta\rangle \text {. }
$$

Thus, the average total modified propagation path is the same as the average modified path length. The square of the standard deviation, or the variance, may be written as

$$
\begin{aligned}
\sigma_{\text {sd }}^{2}\left(\zeta_{\text {tot }}\right) & =\sigma_{\text {sd }}^{2}(\zeta)-\frac{2 \Gamma^{2}\left(-\frac{1}{4}\right)}{35 \pi^{3 / 2}} \sqrt{a R_{0}^{3}}+\frac{R_{0}^{2}}{4} \\
& \approx 0.011 a R_{0}-0.247 a^{1 / 2} R_{0}^{3 / 2}+0.25 R_{0}^{2} .
\end{aligned}
$$

Thus $\sigma_{\mathrm{sd}}\left(\zeta_{\text {tot }}\right) \approx R_{0} / 2$ (since $R_{0} \gg a$ ), or that the variation in the total propagation path is dominated by the distance traveled by the incident plane wave. This result may be expected, as the modified length increases much slower than the true length used with the plane wave, and therefore adds little to the total modified propagation path.

Since the modified length may be considered negligible relative to the incident wave propagation distance, it may be 
neglected in calculating the nonlinear distortion measured at the center of the circle of scatterers. This distortion may then be readily estimated as a function of time. Two scattered signals will be measured simultaneously if their total physical propagation lengths, source to scatterer plus scatterer to center, are equal (neglecting faster or slower propagation speeds due to nonlinearity). For a plane wave source this occurs for all scatterers along parabolic edges described by

$$
x=\frac{y^{2}-\left(d-d_{0}\right)^{2}}{2\left(d-d_{0}\right)},
$$

where $(x, y)$ is the coordinate relative to the center of the circle, $d$ is the total physical propagation distance, and $x=d_{0}$ is the reference plane of the incident plane wave (see Fig. 6 for a visual representation). Equation (29) is parameterized by $d$. Since the wave speed between scatterers is the constant $c_{0}$ (again, neglecting nonlinear variations in sound speed) the total physical propagation distance may be written in terms of the arrival time $t$ as $d=c_{0} t$, where $t=d_{0} / c_{0}$ refers to the time when an incident phase plane passes through the center of the circle. For convenience of notation, let $\eta \equiv c_{0} t-d_{0}$. The propagation distance of the incident wave averaged along the parabola (i.e., not including the propagation from the scatterer to the center or measurement location) represents the characteristic propagation distance that is important for the nonlinear distortion measured at the center, and may be calculated as

$$
\begin{aligned}
\left\langle d_{0}-x\right\rangle= & d_{0}+\frac{\eta}{2}-\frac{\eta}{8} \\
& \times \frac{y_{0}\left(1+2 y_{0}^{2} / \eta^{2}\right) \sqrt{1+y_{0}^{2} / \eta^{2}}-\eta \sinh ^{-1}\left(y_{0} / \eta\right)}{y_{0} \sqrt{1+y_{0}^{2} / \eta^{2}}+\eta \sinh ^{-1}\left(y_{0} / \eta\right)},
\end{aligned}
$$

where

$$
y_{0}=\sqrt{2 \eta R_{0}-\eta^{2}}
$$

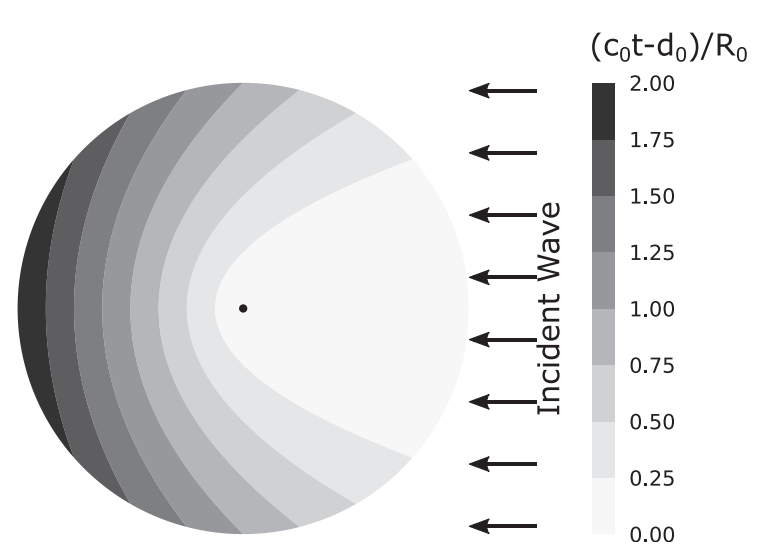

FIG. 6. Arrival time of signal as a filled contour map of where on the circle of scattering cylinders the signal comes from. The dot at the center of the circle is the measurement site. The circle radius is $R_{0}$, the distance of the incident plane wave reference plane is $d_{0}$, and the ambient small-signal wavespeed is $c_{0}$. is the $y$ coordinate at which the edge of the circle intersects the parabola. This complicated expression may be approximated rather well by a linear expansion from $\eta=2 R_{0}$, given by

$$
\left\langle d_{0}-x\right\rangle \approx d_{0}+\frac{2 \eta-R_{0}}{3} .
$$

Both the exact and linear approximation of the mean propagation distance of the incident wave are shown in Fig. 7 assuming $d_{0}=R_{0}$. Varying $d_{0}$ induces a vertical translation of the plot, but has no other effect. It is important to remember that scattering close to forward-scattering is not well represented by geometrical acoustics, and so actual measurements may not follow the above trend for $c_{0} t-d_{0} \ll R_{0}$. An interesting feature in Fig. 7 is that the mean propagation distance is actually less than $d_{0}$ for early measurements since much of the scattering occurs along the direct route. In fact, the mean propagation distance is equal to $d_{0}$ close to $c_{0} t=d_{0}+R_{0} / 2$. Another interesting feature is that the monotonic increase in mean propagation distance of the incident wave with respect to time suggests that later scattering events will be more distorted than earlier ones. Thus, if the incident plane wave were a short pulse, the measured waveform would be expected to be a series of pulses that become increasingly more distorted as time progresses. For comparison, if there were no scatterers present, the measured waveform would be expected to be a single pulse with the mean propagation distance of the incident wave being trivially equal to $d_{0}$.

An explicit example may be helpful. Consider the incident pulse described in Sec. IID with a source location at $x$ $=R_{0}$, where $R_{0}=100 a=10 \mathrm{~m}$. To approximate the conditions under which the above analysis holds, i.e, dilute concentration of cylinders uniformly distributed about the circle of scatterers, consider $N=100$ cylinders placed in a Fibonacci lattice, such that the distance to the center and angle relative to the $x$ axis of the $k$ th cylinder may be written as

$$
r_{k}=R_{0} \sqrt{\frac{k+1 / 2}{N}}, \quad \theta_{k}=\frac{2 \pi k}{(1+\sqrt{5}) / 2},
$$

where $k \in\{0,1,2, \ldots, N-1\}$. In this case we may write

$$
\hat{d} \approx \sqrt{\frac{N}{\pi R_{0}^{2}}} \approx 56 \mathrm{~cm} \gg a=10 \mathrm{~cm} .
$$

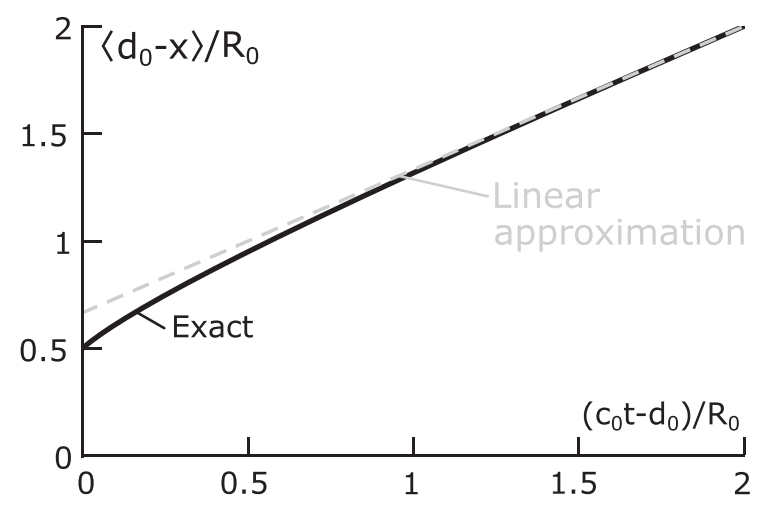

FIG. 7. Normalized mean propagation distance of the incident wave as a function of normalized time. For this plot $d_{0}=R_{0}$. 


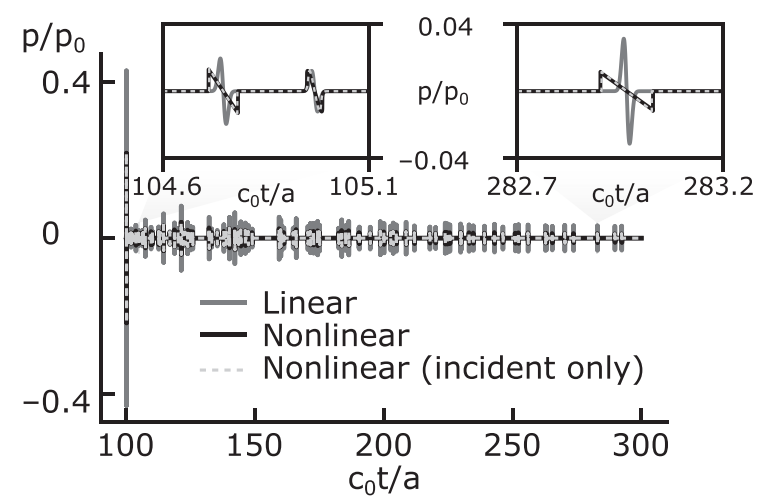

FIG. 8. Waveforms predicted to be measured as a function of time at the center of a circle of scatterers as a function of time using linear propagation, nonlinear propagation as described in the main text, and nonlinear propagation for the incident wave only with linear propagation thereafter. The inset graphs show close-ups of portions of the waveform early (left) and late (right) in the measured signal.

Figure 8 shows the waveform predicted to be measured at the center of the circle assuming linear propagation, assuming nonlinear propagation, and assuming nonlinear propagation until after a scattering event (i.e., only for the incident wave). As may be seen in the figure, the overall trend of the predictions is the same: a large peak arrives at the beginning of the signal followed by a train of much smaller peaks. The first peak is a combination of the incident wave and the scattered signals that scatter close to the $x$ axis. As time progresses, the scattered signals begin to spread out in time. Since the incident pulse has a relatively small time scale the scattered signals do not overlap much, but when they do overlap linear superposition holds for both linear and nonlinear propagation cases [see the discussion around Eq. (23), above]. As may be seen in the close-ups inset in Fig. 8, the two nonlinear cases are nearly identical, demonstrating the conclusion found above that the nonlinear evolution of the incident wave dominates the overall nonlinear evolution. Given the results in Sec. IIC, it should be noted that these results are only valid for signals where the shock formation distance is much larger than the cylinder radius. The nonlinear cases exhibit many shocks. Since the radius of the circle of scatterers is about four times the characteristic shock formation distance, these shocks are expected. In the inset figure from early in the predicted waveform (left) the shocks are relatively high amplitudes and are closely spaced compared with features appearing later in the predicted waveform (right inset figure). The lower amplitude of the later shocks is due to the incident wave having propagated farther and thus enabling additional nonlinear distortion. The greater spread of the shocks later in the predicted waveform come from the fact that there are fewer propagation paths with the later arrival times, as represented graphically in Fig. 6.

\section{SUMMARY}

An extension of traditional geometric acoustics to account for nonlinearity has been used to describe scattering of high-frequency, high-amplitude acoustic waves by rigid cylinders. The importance of incorporating nonlinearity (as measured by the ratio of the plane to scattered ray shock formation distances) in the scattered field was shown to be angle-dependent, with incorporation being most important in the back-scattered angles and least important in the forward-scattering angles. The impact of scattering by many cylinders was also discussed, and it was shown that, for signals where the shock formation distance is much larger than a cylinder radius, the nonlinear evolution of the incident wave is far more important to the measured signal than the nonlinear evolution of the individual scattered rays. These results were demonstrated in waveform analyses using an example pulsed waveform.

\section{ACKNOWLEDGMENTS}

The authors would like to thank Gregory W. Lyons (U.S. Army Engineer Research Development Center) for his thoughtful contributions in reviewing the manuscript, as well as the anonymous reviewers for their helpful suggestions. This study was conducted for the Office of the Assistant Secretary of the Army for Acquisition, Logistics, and Technology [ASA(ALT)] under Research, Development, Test, and Evaluation Program Element 611102T2400, Signature Physics and Terrain State Basic Research. Permission to publish was granted by Director, Cold Regions Research and Engineering Laboratory.

${ }^{1}$ T. F. W. Embleton, "Scattering by an array of cylinders as a function of surface impedance," J. Acoust. Soc. Am. 40, 667-670 (1966).

${ }^{2}$ J. V. Sánchez-Pérez, D. Caballero, R. Mártinez-Sala, C. Rubio, J. Sánchez-Dehesa, F. Meseguer, J. Llinares, and F. Gálvez, "Sound attenuation by a two-dimensional array of rigid cylinders," Phys. Rev. Lett. 80, 5325-5328 (1998).

${ }^{3}$ R. J. Hughes, J. A. S. Angus, T. J. Cox, O. Umnova, G. A. Gehring, M. Pogson, and D. M. Whittaker, "Volumetric diffusers: Pseudorandom cylinder arrays on a periodic lattice," J. Acoust. Soc. Am. 128, 2847-2856 (2010).

${ }^{4}$ P. Chobeau, G. Guillaume, J. Picaut, D. Ecotière, and G. Dutilleux, "A transmission line matrix model for sound propagation in arrays of cylinders normal to an impedance plane," J. Sound Vib. 389, 454-467 (2017).

${ }^{5}$ I. B. Abbasov, "Study of the second harmonics in nonlinear scattering of interacting acoustic waves by a rigid cylinder," Acoust. Phys. 47, 633-639 (2001).

${ }^{6} \mathrm{R}$. T. Beyer, Nonlinear Acoustics (Acoustical Society of America, New York, 1997).

${ }^{7}$ G. T. Silva and A. Bandeira, "Difference-frequency generation in nonlinear scattering of acoustic waves by a rigid sphere," Ultrasonics 53, 470-478 (2013).

${ }^{8}$ A. D. Pierce, Acoustics: An Introduction to Its Physical Principles and Applications (Acoustical Society of America, Woodbury, NY, 1989).

${ }^{9}$ J. B. Keller, "Geometrical acoustics. I. The theory of weak shock waves," J. Appl. Phys. 25, 938-947 (1954).

${ }^{10}$ K. O. Friedrichs and J. B. Keller, "Geometrical acoustics. II. Diffraction, reflection, and refraction of a weak spherical or cylindrical shock at a plane interface,” J. Appl. Phys. 26, 961-966 (1955).

${ }^{11}$ C. L. Morfey, F. D. Cotaras, and A. D. Pierce, "Propagation in inhomogeneous media (ray theory)," in Nonlinear Acoustics, edited by M. F. Hamilton and D. T. Blackstock (Acoustical Society of America, New York, 2008), pp. 343-376.

${ }^{12}$ F. B. Jensen, W. A. Kuperman, M. B. Porter, and H. Schmidt, Computational Ocean Acoustics, 2nd ed. (Springer Science \& Business Media, New York, 2011). 
${ }^{13}$ G. Deschamps, "Ray techniques in electromagnetics," Proc. IEEE 60, 1022-1035 (1972).

${ }^{14}$ D. T. Blackstock, M. F. Hamilton, and A. D. Pierce, "Progressive waves in lossless and lossy fluids," in Nonlinear Acoustics, edited by M. F. Hamilton and D. T. Blackstock (Acoustical Society of America, New York, 2008), pp. 65-150.
${ }^{15}$ M. F. Hamilton, "Sound beams," in Nonlinear Acoustics (Acoustical Society of America, New York, 2008), pp. 233-262.

${ }^{16}$ H. Primakoff, M. J. Klein, J. B. Keller, and E. L. Carstensen, "Diffraction of sound around a circular disk," J. Acoust. Soc. Am. 19, 132-142 (1947). 


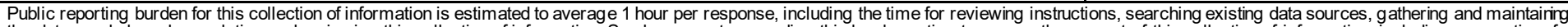

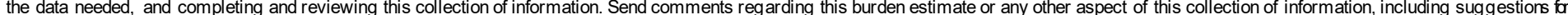

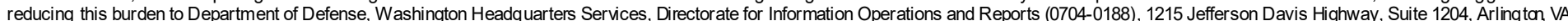

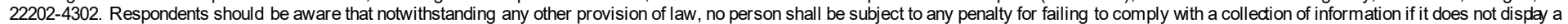
currently valid OMB control number. PLEASE DO NOT RETURN YOUR FORM TO THE ABOVE ADDRESS.

\begin{tabular}{|l|l|l}
\hline $\begin{array}{l}\text { 1. REPORT DATE (DD-MM-YYYY) } \\
\text { October } 2020\end{array}$ & $\begin{array}{l}\text { 2. REPORT TYPE } \\
\text { Final }\end{array}$ & 3. DATES COVERED (FrOm - To) \\
\hline 4. TITLE AND SUBTITLE & 5a. CONTRACT NUMBER
\end{tabular}

Geometric-Acoustics Analysis of Singly Scattered, Nonlinearly Evolving Waves by

Circular Cylinders

5a. CONTRACT NUMBER

5c. PROGRAM ELEMENT NUMBER

$611102 \mathrm{~T} 2400$

6. AUTHOR(S)

Micha el B. Muhlestein and Carl R. Hart

5d. PROJECT NUMBER

$\mathrm{AB} 2$

5e. TASK NUMBER

5f. WORK UNIT NUMBER

7. PERFORMING ORGANIZATION NAME(S) AND ADDRESS(ES)

Cold Regions Research and Engineering La boratory

8. PERFORMING ORGANIZATION REPORT NUMBER

ERDC/CRREL MP-20-16

U.S. Army Engineer Research and Development Center

72 Lyme Road

Hanover, NH 03755

9. SPONSORING / MONITORING AGENCY NAME(S) AND ADDRESS(ES)

US Army Corps of Engineers

Office of Assistant Secretary of the Army for Acquisition, Logistics, \& Technology

Wa shington, DC 203 14-1000

10. SPONSOR/MONITOR'S ACRONYM(S)

11. SPONSOR/MONITOR'S REPORT NUMBER(S)

\section{DISTRIBUTION / AVAILABILITY STATEMENT}

Approved for public release; distribution is unlimited.

\section{SUPPLEMENTARY NOTES}

AMSCOCode 611102 AB200

This article was originally published in the Journal of the Acoustic Society of America, June 2020.

\section{ABSTRACT}

Geometric acoustics, or a coustic ray theory, is used to analyze the scattering of high-amplitude acoustic waves incident upon rigid circular cylinders. Theoretical predictions of the nonlinear evolution of the scattered wa ve field are provided, as well a s measures of the importance of a ccounting for non linearity. An a nalysis of scattering by many cylinders is also provided, though the effects of multiple sca ttering a re not considered. Provided the characteristic nonlinear distortion length is much larger than a cylinder ra dius, the nonlinear evolution of the incident wa ve is shown to be of much grea ter importance to the overall evolution than the nonlinear evolution of the individual scattered waves.

\section{SUBJECT TERMS}

Sound, Sound-waves, Shock waves, Nonlinear waves, Cylinders

\begin{tabular}{|l|c|c|c|l|l|}
\hline \multicolumn{2}{|l|}{ 16. SECURITY CLASSIFICATION OF: } & $\begin{array}{l}\text { 17. LIMITATION } \\
\text { OF ABSTRACT }\end{array}$ & $\begin{array}{l}\text { 18. NUMBER } \\
\text { OF PAGES }\end{array}$ & $\begin{array}{l}\text { 19a. NAME OF RESPONSIBLE } \\
\text { PERSON }\end{array}$ \\
\cline { 1 - 2 } $\begin{array}{c}\text { a. REPORT } \\
\text { Unclassified }\end{array}$ & $\begin{array}{c}\text { b. ABSTRACT } \\
\text { Unclassified }\end{array}$ & $\begin{array}{c}\text { c. THIS PAGE } \\
\text { Uncla ssified }\end{array}$ & SAR & 12 & $\begin{array}{l}\text { 19bea code) } \\
\text { area conO NUMBER (include }\end{array}$ \\
\hline
\end{tabular}

